\title{
Immanuel Wallerstein: globalización de la economía-mundo capitalista. Perspectiva de largo plazo
}

Crisóstomo Pizarro Contador.

Fondo de Cultura Económica, Santiago, 2016.

106 páginas.

Explicar y sintetizar el monumental pensamiento teórico de Immanuel Wallerstein en un discreto libro de bolsillo ha sido el reciente aporte del Dr. Crisóstomo Pizarro, publicado por el Fondo de Cultura Económica Chile, en julio de 2016.

El autor de esta útil obra corre con ventaja al interpretar los escritos de Wallerstein, puesto que el teórico fue su director de tesis en la Universidad de Columbia, mientras cursaba el Master of Arts en Sociología. El Dr. Pizarro ha logrado construir en un pequeño, pero muy completo libro, una interpretación sistémica del pensamiento teórico de Wallerstein, en especial, sobre los mecanismos y la evolución histórica del sistema-mundo capitalista, vinculándolo con una mirada de largo plazo, tomando el fenómeno de la globalización como punto de fuga de su análisis.

El texto pareciera incitar una lectura interpretativa del fenómeno de la globalización, a partir del pensamiento de Immanuel Wallerstein. Sin embargo, a las pocas páginas se evidencia el verdadero objetivo de la obra: el estudio de la evolución del capitalismo histórico (p. 11), considerando la globalización como una parte concreta de aquella evolución histórica.

La obra es presentada por el mismo Immanuel Wallerstein, quien aclara que los alcances del libro, en términos generales, están orientados a contribuir a la reflexión y el debate en el escenario mundial, respecto del devenir del sistema-mundo capitalista en el mediano y largo plazo. Es 
decir, el libro se sitúa en una fase inicial de transformación mundial, donde se discute, en palabras de Wallerstein, cuál será el sistema que remplazará al moribundo sistema capitalista (p. 11). Según asegura el teórico en la presentación del libro reseñado, "esta disputa se presenta como una elección global entre un sistema no capitalista, pero que conservaría los peores elementos del capitalismo -jerarquía, explotación y polarización- y un nuevo sistema que será relativamente democrático e igualitario, y que nunca ha existido anteriormente" (p. 10).

Así, la obra inicia problematizando la situación actual de transformación y cambio del sistema mundial, entregándonos algunos antecedentes históricos de la globalización y analizándolos posteriormente, a la luz del pensamiento de Wallerstein, siempre haciendo, por supuesto, la distinción entre lo expresado por el teórico y lo interpretado por el Dr. Pizarro.

El libro se divide en nueve breves secciones, en las cuales se construye una interpretación explicativa del proceso histórico evolutivo de capitalismo mundial, apoyado en la fase final por una exacerbación exitosa de la globalización mundial ${ }^{1}$. Es importante señalar que para Wallerstein, en dicho éxito se situaría el fin del sistema-mundo capitalista pues, desde su óptica, el capitalismo va a terminar pronto, pero no terminará por sus contradicciones, sino que lo hará a partir de su profundo éxito histórico ${ }^{2}$.

En la primera sección del libro, en la parte introductoria, el autor aborda la evolución del sistema-mundo capitalista desde el concepto de la Longue Durée 3 , caracterizando a

1 Para una caracterización más general de la explicación de crisis terminal del capitalismo, ver la entrevista a Immanuel Wallerstein: Un système s ècroule aujourd hui sous nos yeux, Alternatives Èconomiques, No 33, Hors-Serie, París, (1997).

2 Aguirre Rojas, C. (2004). Immanuel Wallerstein: critica del sistema-mundo capitalista: estudio y entrevista. Santiago: Lom Ediciones, pp. 92.

3 El concepto de ondas largas "Longue Durée" ha sido trabajado latamente en: Wallerstein, I. (ed.) (2016). Modern World-System in the Longue Durée. New York: Routledge. Sin embargo, el autor explica que el calificativo «largos» designa la cualidad de los procesos de larga duración, que transcurren en períodos que 
la globalización como la tendencia histórica del desarrollo de la economía mundo-capitalista. El autor posiciona el largo siglo XVI como la primera ola de expansión global de la economía-mundo capitalista (Europa Occidental Europa Oriental - América Latina), al mismo tiempo que se estabiliza la economía-mundo capitalista, a partir del desarrollo de una agricultura capitalista y el fracaso de los intentos imperialistas de España y Francia (p. 12). El siglo XVII, según el autor, significó la consolidación de los límites geográficos del mundo. Corresponde a la fase mercantilista del sistema-mundo capitalista, que involucra la incorporación de Rusia, India, África, el Imperio Otomano y los procesos de descolonización de América. En tanto que el siglo XIX se caracteriza por la expansión territorial y la incorporación de nuevos actores al sistema-mundo: China, Japón y Corea. Por último, el análisis del largo siglo XX y XXI es divido por el autor en cuatro etapas: primero, la decadencia de Inglaterra y el surgimiento de la rivalidad entre Estados Unidos y Alemania. Segundo, la victoria de Estados Unidos sobre Alemania. Tercero, la pax americana o Guerra Fría. Y por último, una fase de contracción de la economía-mundo. Esta última etapa es explicada por el autor de acuerdo a la fase B de los llamados ciclos Kondràtieff. En esta primera sección del libro se proponen y grafican dos completas tablas (pp.16-21), realizadas por el autor para caracterizar la evolución histórica del sistemamundo capitalista y explicar las diferencias entre los ciclos Kondràtieff y la Longue Durée.

En la segunda sección, de muy breve desarrollo, titulado Longue Durée y Estructura, el autor utiliza la conceptualización de Fernad Braudel para considerar críticamente la temporalidad de los fenómenos históricos; es así que logra vincular la Longue Durée con el concepto de Estructura. El Dr. Pizarro afirma que la estructura es afectada solo levemente por el transcurso del tiempo y da lugar a una relación estable entre grupos sociales y realidad social ( $\mathrm{p}$. 26). Esta sección se fundamenta por una nota aclaratoria

se extienden más allá del criterio cronológico que se aplica convencionalmente para distinguir un siglo de otro (p. 12). 
hecha por Immanuel Wallerstein al borrador del libro, según aclara posteriormente el autor.

La tercera sección del libro, titulada Intercambio Desigual entre Centro y Periferia, se encarga de explicar el proceso de extracción de plusvalía ${ }^{4}$ por parte de los Estados céntricos respecto de los periféricos, en la constante secuela de la división global del trabajo. Para ello, el Dr. Pizarro utiliza fundamentalmente la obra de Immanuel Wallerstein para explicar las interacciones y transformaciones que han vivido los Estados desde su condición de centro, periferia y/o semiperiferia, considerando para ello las formas de intercambio entre estas tres situaciones y sus características principales. Por ejemplo, el autor asegura que los países periféricos, a diferencia de los centrales, se caracterizan por una organización estatal y una autonomía extremadamente débiles o inexistentes (p. 31). De esta forma, se genera un intercambio en desiguales condiciones.

Para el Dr. Pizarro, la interacción entre Estados centrales, periféricos y semiperiféricos en el sistema interestatal, "se regula por un balance de poder que impide que la división social del trabajo corresponda a una superestructura imperial, esto es la existencia de un único centro político y eje de la división social del trabajo sobre los territorios comprendidos dentro de sus límites geográficos sostenido en una enorme estructura burocrática" (p. 33). En esa sección, se presenta un tabla explicativa sobre la expansión geográfica de la economía-mundo capitalista entre los siglos XVI y XX (pp. 40-41).

La cuarta sección de la obra aborda la relación existente entre las Estructuras del Sistema y los citados ciclos Kondràtieff en sus respectivas fases A y B. Es decir, el autor se preocupa de explicar los comportamientos rítmicos o regularidades en los ascensos y descensos de la economíamundo capitalista, que son el motor de su desarrollo y reproducción. El autor hace una inmejorable analogía

\footnotetext{
$4 \quad$ El autor explica este proceso como el resultado de la transacción entre productos de los países centrales de alto costo salarial y productos de los países periféricos, de bajo consto salarial, produciéndose entre ellos una situación de intercambio desigual (p. 30).
} 
para explicar las fases de los ciclos Kondràtieff, que vale la pena citar. Señala:

La fase A del ciclo Kondràtieff podría compararse con el acto de inhalar oxigeno de las innovaciones en los procesos tecnológicos y formas de organización del capital y el trabajo, inversiones y expansiones; mientras que la fase B consistiría en el acto de exhalar los elementos tóxicos, tales como los productores y líneas de producción ineficientes, y de esta forma entrar en una fase de revitalización (p. 43).

En la quinta sección, el autor aborda el constante balance de poder existente entre la pluralidad de Estados del sistema internacional, que configuran la superestructura del sistema económico y que buscan incansablemente obtener una posición mundial hegemónica. En este sentido, se abordan críticamente los elementos históricos que han permitido la variabilidad temporal de la hegemonía en determinados Estados. El autor distingue en la teoría de Wallerstein, cinco rasgos que caracterizan la posición hegemónica de un país: primero, el liderazgo económico basado en la innovación de los procesos tecnológicos. Segundo, la defensa del liberalismo global, apoyo a las instituciones parlamentarias y restricciones a la arbitrariedad de la burocracia estatal. Tercero, el control sobre las actividades de subsistencia, la pequeña producción mercantil y las rentas procedentes de las diferentes formas de propiedad. Un cuarto rasgo de los países hegemónicos es la distinción entre unidades domésticas proletarias y semiproletarias, de acuerdo a la proporción de los ingresos originados en el trabajo asalariado, en comparación con otras fuentes de ingreso (p. 54). Finalmente, un último rasgo de la hegemonía es el desarrollo del poder militar, capaz de mantener un Estado de guerra constante.

La sección sexta de la obra lleva por título Declive de Estados Unidos y Crisis Global, en la cual el autor aborda analíticamente las perspectivas de Wallerstein, en cuanto a la pérdida de hegemonía de Estados Unidos, afirmado que esta se originaría en su creciente incapacidad para seguir 
manteniendo el liderazgo mundial en innovación tecnológica, producción, comercio y finanzas, acompañadas de una caída del poder político y militar. Esto a partir del deterioro de legitimidad política, utilizada para transferir los costos de la producción a las zonas periféricas de la economía-mundo capitalista.

A continuación, la séptima sección aborda las contradicciones entre acumulación de capital y democratización. En ella, el autor reflexiona sobre los alcances de la etapa terminal del capitalismo mundial, atribuyéndole merecida importancia a los micro procesos sociales que se desarrollan al margen del sistema de acumulación, en forma de minorías descontentas y bajo la búsqueda de derechos sociales. El autor identifica y problematiza variados temas actuales, tales como la migración, los conceptos actuales de ciudadanía, la crisis de legitimad del Estado, etc., y los vincula para construir una explicación subyacente a la fase actual de sistema-mundo capitalista.

La octava sección del libro comienza a sintetizar la idea de crisis terminal del sistema. Se aborda reflexivamente desde la caída de la geocultura del liberalismo, hasta los procesos recientes de apertura mundial a la protección de los derechos humanos, como nuevo paradigma de la humanidad, símil de lo que significó para los países periféricos el principio de autodeterminación de los pueblos. El Dr. Pizarro entiende dicho proceso a la luz de los argumentos de Wallerstein ${ }^{5}$, quien afirma que la crisis de la ideología liberal se basa principalmente en la incompatibilidad del liberalismo y el capitalismo. Una cita de Wallerstein utilizada por el autor explica de mejor manera esta incompatibilidad.

La contradicción intrínseca de la ideología liberal es total. Si todos los seres humanos tienen iguales derechos y todos los pueblos tienen iguales derechos, nosotros

\footnotetext{
El autor utiliza, para esta sección, los trabajos de Wallerstein que abordan dicho problema: After Liberalism, New York: The New Press,1995; Wallerstein, I. (1990). Culture as the ideological battleground of the modern world-system. Theory, culture \& society, 7(2), pp. 31-55; Wallerstein, I. (1991). Geopolitics and geoculture: Essays on the changing world-system. Cambridge University Press.
} 
no podemos mantener un sistema "inigualitario", que será un rasgo propio de la economía-mundo capitalista. Si esto se admite abiertamente, la economía capitalista mundial ya no parecerá legítima a las clases excluidas de sus beneficios o severamente perjudicadas. La crisis es total; el dilema es total. Sufriremos las consecuencias en la próxima mitad del siglo. Sin embargo, colectivamente resolveremos esta crisis, cualquiera sea el nuevo sistema histórico que construyamos (p. 75).

La novena y última sección es, a juicio personal, la más importante de todas, comprendiendo obviamente que todas las secciones son de extrema necesidad para lograr la síntesis esperada por el autor. En esta sección se plantean las formas y pretensiones teóricas realizadas por Wallerstein, para proyectar la transformación del sistema y su desplazamiento por uno nuevo. Uno sin antecedentes históricos claros; uno de características desconocidas para nosotros, pero que debe ser alcanzado a través de un pensamiento utopístico, entendido este como la reflexión y el debate de los dilemas actuales del orden democrático que se desea construir (p. 77). En esa misma línea, el pensamiento utopístico debe converger con la concepción de identidad grupal múltiple, como fase superior del despertar social y colectivo de la humanidad. Aquella humanidad que construirá el nuevo modelo histórico que desplazará al sistema capitalista mundial. Es por ello que la seudo-etapa terminal del capitalismo, la globalización actual, también ha generado espacios para desarrollar este pensamiento utopístico y la identidad grupal.

El Dr. Pizarro identifica como uno de esos espacios de proyección y cambio de realidad social, al Foro Social Mundial. Frente a este organismo, el autor reflexiona sobre sus errores de trayectoria y posibles propuestas, como alternativa de transformación estructural y plataforma de discusión utopística para una nueva realidad mundial.

Finalmente, la obra termina con una breve nota que aborda sintéticamente lo esperado por el autor al escribir el libro. En especial, la idea de cambio constante y la evidente certeza sobre la profunda crisis del capitalismo, 
su etapa terminal actual y sus proyecciones como modelo obsoleto, que será desplazado en un futuro medio por un nuevo modelo de desconocidas características.

En conclusión, el libro reseñado es sin duda una completa obra de síntesis teórica, basada principalmente en los postulados de Immanuel Wallerstein, pero que a través de las interpretaciones expertas de su autor se convierte en un instrumento de diálogo y debate sobre la evolución histórica de la economía-mundo capitalista. Su estructura es sencilla y de rápida lectura. El nivel de síntesis efectuado para explicar las macroteorías económicas es indiscutiblemente impresionante. Su lectura logra ilustrar al lector menos próximo a la temática, aproximándolo introductoriamente a las problemáticas del pensamiento de Immanuel Wallerstein. Es, sin duda, una lectura recomendada para comprender eficazmente el desarrollo histórico-crítico del capitalismo mundial y sus proyecciones en el siglo XXI.

Hugo J. Castro Valdebenito

Centro de Estudios y Asistencia Legislativa

Pontificia Universidad Católica de Valparaíso 